\title{
SUMMER PEAK, WINTER MINIMUM, AND GROWTH IN THE DEMAND FOR ONLINE WEATHER SERVICES IN AUSTRALIA
}

\author{
by Scott B. Power and François Delage
}

G oogle provides an online tool (http:// google.com/insights/search/\#) to examine the frequency of Google searches for user-specified words or terms back to 2004. Monthly totals for each year are provided and searches made in numerous countries can be investigated. The purpose of this note is to document a prominent annual cycle and growth in the number of searches made for the terms "weather," "weather forecast," the "Bureau of Meteorology," and related terms.

Figure 1 shows the number of times Australians online conducted Google searches for (a) "weather" and "BoM"; (b) "weather forecast," "Sydney weather," and "Melbourne weather"; and (c) "Bureau of Meteorology" and "weather radar" each month, from January 2004 to December 2010. A pronounced annual cycle is evident in all searches, with most searches conducted during summer and fewest searches in winter. The summer peak in
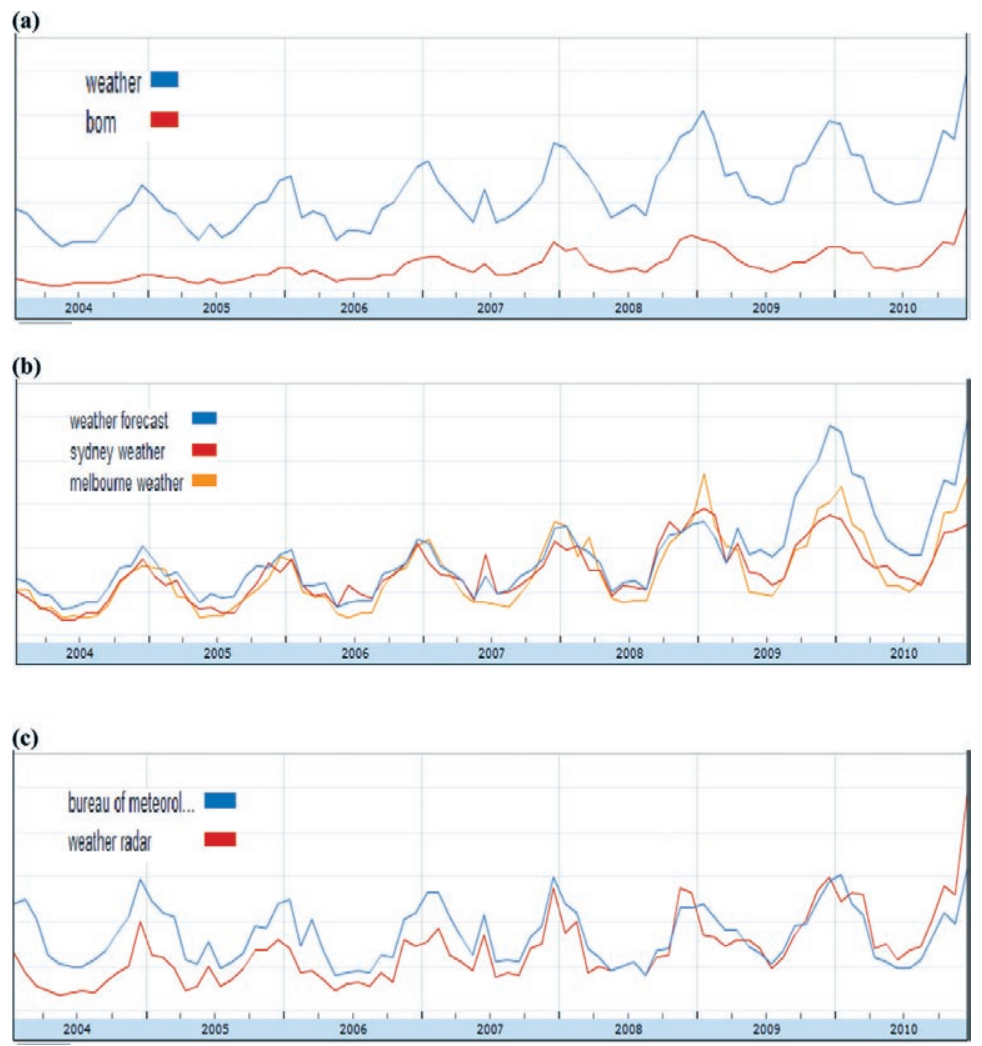

FIG. I. Relative frequency of Google searches

in Australia for key words indicated: (a) "weather" and "BoM"; (b) "weather forecast," "Sydney weather," and "Melbourne weather"; and (c) "Bureau of Meteorology" and "weather radar." Search numbers vary greatly between plots and so plots have been rescaled in order that the variability and trends can be easily seen. The average numbers of (normalized) searches over the full period for these terms are as follows: (a) "weather" (44) and "BoM" (II); (b) "weather forecast" (9), "Sydney weather" (5), and "Melbourne weather" (5); (c) "Bureau of Meteorology" (2) and "weather radar" (1.6). The values here indicate that "weather" for example, is a much

AFFILIATIONS: POWER AND DELAGE-Centre for Australian Weather and Climate Research, Bureau of Meteorology, Melbourne, Australia

CORRESPONDING AUTHOR: Scott B. Power, Centre for Australian Weather and Climate Research, Bureau of Meteorology, GPO Box 1289, Melbourne, Australia

E-mail: s.power@bom.gov.au

DOI:10.|I75/20IIBAMS3II3.I

C2011 American Meteorological Society more common search term than "weather forecast," with a ratio of $44: 9$. The vertical scale in all plots is a normalized value obtained by multiplying all monthly search totals by $100 / N_{\max }$, where $N_{\max }$ is the maximum number of searches that occurred in any month during the entire period (i.e., from Jan 2004 to Dec 2010), for any of the search terms used in each plot. In (a), for example, $N_{\max }$ occurred for "weather" in Dec 2010, and so it is set to a value of 100 . The ratio of search totals is 57:26. Search frequencies for "BoM" and "weather" are presented separately in (a) because they dwarf the search frequencies for the terms in (b). Each year is separated by the faint vertical lines in each plot. 
(a)

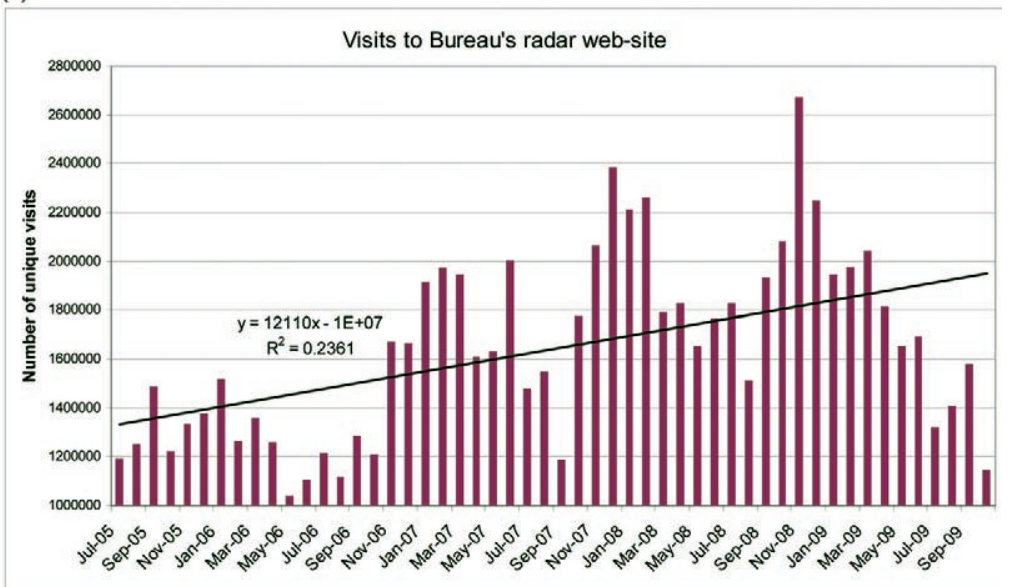

(b)

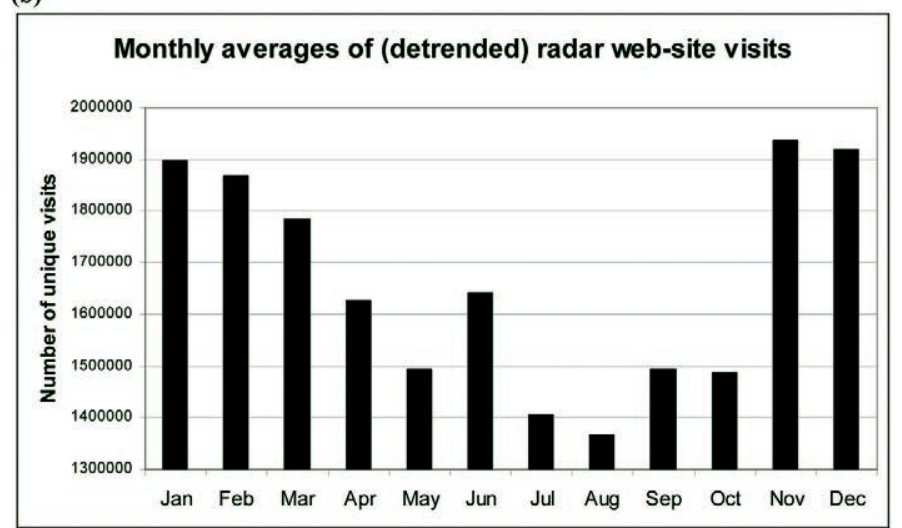

FIG. 2. (a) Frequency of visits to the Bureau of Meteorology's radar Web site as monitored by the Bureau, and (b) the corresponding annual cycle of detrended visit frequencies. Linear trend shown in (a). for example, the search frequency in summer is about $180 \%$ greater than the corresponding value in winter!

The Bureau of Meteorology also monitors the frequency with which people visit its Web pages. The frequency of visits to the Bureau's radar Web pages is depicted in Fig. 2. A similar annual cycle and growing trend are evident in these independently derived statistics.

What causes the annual cycle in these search frequencies and Web site visits? Is it linked to the frequency of severe weather-related events? To test this hypothesis, we produced search statistics for "bushfire," "flood," and "tropical cyclone." The evolution of the search frequencies for these three terms (Fig. 3) does not bear any resemblance to the cyclic plots in Fig. 1-the time series are much more erratic; there is a weak tendency for a summer peak in searches for "bushfire" and "tropical cyclone," but the seasonal cycle makes a much smaller contribution to the variability than for "weather," etc. There is no summer peak in searches for "flood" at all. So searches for severe weather-related events do not account for the prominence and regularity of the annual cycles in Figs. 1 and 2. "weather" was previously noted in a 2009 online article on www.news.com.au ("Weather Web Searches Take on Sex," 20 November 2009). Here we see that similar peaks are evident in terms more directly linked with online weather services. In searches for "Bureau of Meteorology,"

FIG. 3. Relative frequency of Google searches in Australia for (a) "tropical cyclone" and "flood," and (b) "bushfire." Note the large peak associated with "Black Saturday" (when eastern Australia endured record heat and deadly bushfires) in Feb 2009 and the record peak in "flood" searches during Dec 2010, which coincided with record-high rainfall in Queensland and widespread flooding across eastern Australia. Average normalized searches for "flood" and "bushfire" equal one, while the equivalent number for "tropical cyclone" is much less than one. (a)

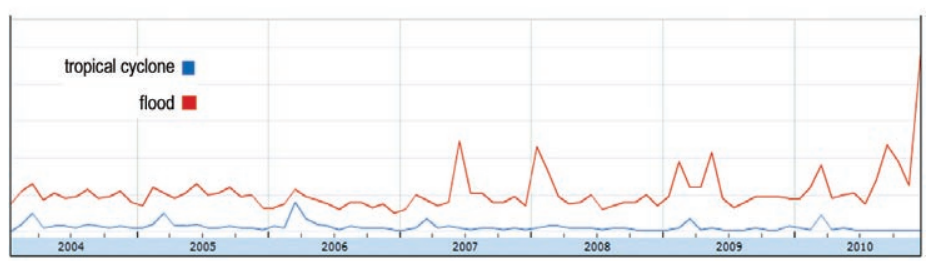

(b)

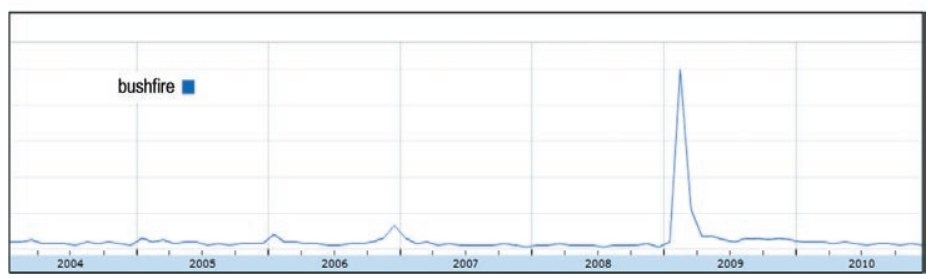

Instead, the seasonal cycle in searches for "weather forecast," etc., might be due to Australians generally having more interest in outdoor, 
weather-impacted activities during summer. This is supported by the fact that very similar pronounced annual cycles are evident in searches for such terms as "holiday," "BBQ," "outdoor," and "boat" (Fig. 4). According to the Bureau's South Australian office director, Andrew Watson: "This is consistent with my impression that the higher volume of enquiry happens during the warmer months, given the

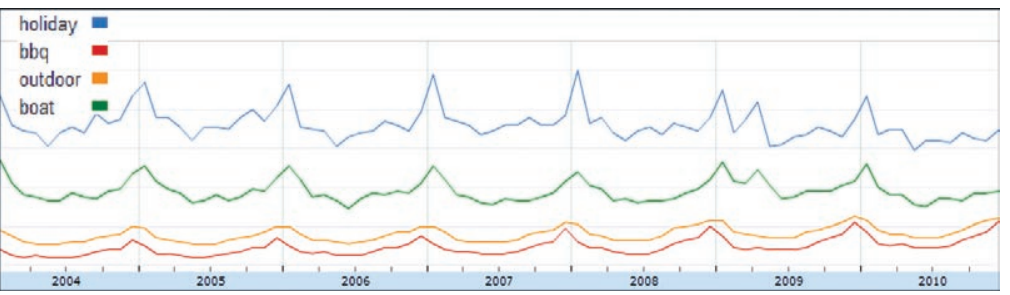

FIG. 4. Relative frequency of Google searches in Australia for "holiday," "BBQ," "outdoor," and "boat." Average normalized searches for these terms are 16, 2, 3, and 8, respectively.

significance of summer weather on the increased variety of outdoor activities." While plausible, further research is needed to confirm that participation in outdoor activities is in fact the main reason for the annual cycles evident in Figs. 1 and 2.

The high volume of online searches for weatherrelated information in Australia is illustrated in Fig. 5, where search frequencies for "weather" are compared with search frequencies for words that loom large in Australian life. Figure 6 shows that searches for "sex" and "weather" are much more frequent than searches for "football," "beer," and "religion." The plot also shows, as highlighted previously in the 2009 www.news.com.au article, that Australians online have searched more frequently for "weather" than they did for "sex" in recent years. The same is also true for the United Kingdom, the United States, Canada, and New Zealand (not shown).

Most of the time series of search frequencies for "weather" and related terms also display positive trends. Yet trends in searches for "outdoor," etc., in Fig. 4 are either absent, negative, or much smaller as a fraction of the

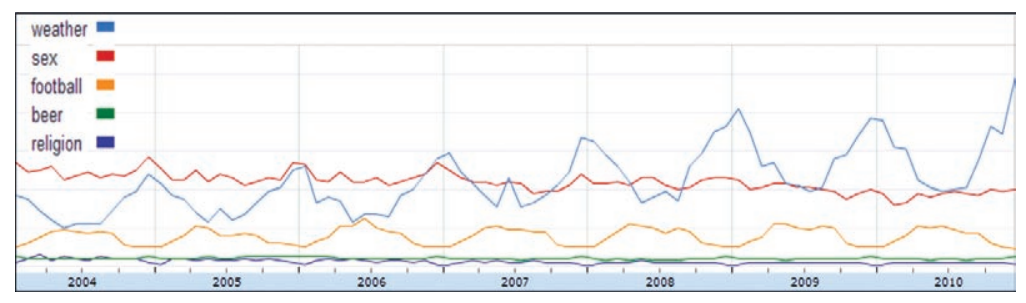

FIG. 5. Relative frequency of Google searches in Australia for "weather," "sex," "football," "beer," and "religion." Average normalized searches for these terms are $44,44,16,4$, and 2 , respectively. variability evident. What causes the growing trend in weather-related searches if it is not a growth in "outdoor"-related searches? While more research beyond the scope of this short news piece is required to address this question, we will conclude by speculating on some of the possible reasons.

While Australia's population has grown from 20,127,400 in 2004 (World Bank data) to an estimated 22,336,133 in May 2010 (Australian Bureau of Statistics data), this would be expected to drive similar trends in searches for "weather," "outdoor," or any other searches, for that matter. Similarly, the

outside or traveling is increasing, and this is likely contributing to the large growth in the frequency with which Australians seek online weather services. This growth is expected to continue in the future as more people take up these mobile technologies and as additional related technologies (e.g., iPads with weather service applications) emerge. Part of the growth is also likely due to an increase in the number of weather radars around the country and an increase in the availability of this information on the Bureau's Web site. 